\title{
The "Battle of the Sexes": A Genetic Model with Limit Cycle Behavior
}

\author{
J. Maynard SMith ${ }^{*}$ and Josef Hofbauer ${ }^{\dagger}$ \\ * School of Biological Sciences, University of Sussex, \\ Falmer, Brighton, Sussex BNI 9QG, England, and \\ ${ }^{\dagger}$ Institut für Mathematik, Universität Wien, \\ Strudlhofgasse 4, A-1090 Wien, Austria
}

Received August 30, 1985

\begin{abstract}
A two-locus genetic model, based on Dawkins' "sex war" game, with the fitness of the genotypes at each locus depending on the gene frequencies at the other, is shown to give rise to a stable limit cycle. The mathematical analysis involves averaging techniques and elliptic integrals. (C) 1987 Academic Press, Inc.
\end{abstract}

\section{INTRODUCTION}

This article describes a simple two-locus genetic model that gives rise to a stable limit cycle. The origin of the model is Dawkins' (1976) "sex war" game, a simple evolutionary game describing the parental investment conflict. In this game males have two possible strategies, faithful $\left(E_{1}\right)$ or philanderer $\left(E_{2}\right)$, and females may play the strategies fast $\left(F_{1}\right)$ or coy $\left(F_{2}\right)$. Assuming +15 as the reward for having a child (to both parents), -20 as the total cost of raising the offspring, and -3 for the prolonged courtship coy females insist on, Dawkins obtained the following payoff matrices for males and females $\left(a_{i j}\right.$ and $b_{j i}$ denote the respective payoffs for an $E_{i}$ male and an $F_{j}$ female after their encounter)

$$
A=\left(\begin{array}{rr}
5 & 2 \\
15 & 0
\end{array}\right), \quad B=\left(\begin{array}{rr}
5 & -5 \\
2 & 0
\end{array}\right) .
$$

This bimatrix game has a totally mixed Nash equilibrium at $\left(\frac{5}{8}, \frac{3}{8}\right)$ and $\left(\frac{1}{6}, \frac{5}{6}\right)$ (see Dawkins, 1976; Maynard Smith, 1982; Schuster \& Sigmund, 1981). Despite Dawkins' claim, however, this is not an evolutionarily stable state (ESS). In fact, Selten (1980) has shown in general that in asymmetric conflicts, only pure strategies can be evolutionarily stable. See also the discussion in Hofbauer (1987) about what an ESS means in asymmetric conflicts. The "battle of the sexes" is therefore one of the simplest games 
without an ESS. For such games the static, game-theoretic approach is not very satisfactory: it can tell us there is no ESS, but to analyze the behavior away from equilibrium we need to introduce dynamics.

It turns out, however, that the standard continuous-time game dynamics, as studied, e.g., by Zeeman (1980), may be generalized in (at least) two different ways to asymmetric conflicts: the one, see Schuster and Sigmund (1981), gives rise to conservative oscillations, the other, see Maynard Smith (1982), Appendix J, and Hofbauer (1987), makes the equilibrium asymptotically stable. With discrete time, moreover, the stationary solution would always be unstable (Eshel \& Akin, 1983).

The first type of dynamics is also relevant for the analysis of our new model in Section 2. With $x_{i}, y_{j}$ denoting the relative frequencies of the strategies $E_{i}, F_{j}$, respectively, and the basic assumption

$$
\begin{aligned}
\frac{\dot{x}_{i}}{x_{i}} & =\text { growth rate of } E_{i} \\
& =\text { average payoff for } E_{i}-\text { average payoff for males } \\
& =(A \mathbf{y})_{i}-\mathbf{x}^{t} A \mathbf{y},
\end{aligned}
$$

Schuster and Sigmund (1981) derive the equations

$$
\begin{aligned}
& \dot{x}=x(1-x)(2-12 y) \\
& \dot{y}=y(1-y)(-5+8 x)
\end{aligned}
$$

with $x=x_{1}=1-x_{2}$ being the frequency of faithful males and $y=y_{1}=1-y_{2}$ being the frequency of fast females. They show that

$$
V=x^{5}(1-x)^{3} y^{2}(1-y)^{10}
$$

is a constant of motion, so that all solutions oscillate around the Nash equilibrium, which also represents the time-average of any orbit. (Note the strong analogy to the Lotka-Volterra predator-prey equations.)

These dynamic models have one drawback, however: they ignore sex. The derivation in (2) assumes implicitly that individuals are haploid and hence males and females reproduce asexually; biologically this is nonsense. Thus there is a need for a genetic model which takes into account the sexual interaction between males and females. One such diploid model was studied by Bomze et al. (1983), again using continuous time and leading to similar conservative oscillations. As this lacks structural stability too, a more natural model would be to consider a diploid model with separated generations. 


\section{THE MODEL}

We consider two loci, with two alleles at each locus. The $A$-locus, with alleles $A$ and $a$, regulates male behavior: $A \rightarrow$ strategy $E_{1}$ (=faithful), $a \rightarrow E_{2}$ (=philanderer), and is not expressed in females. More precisely, we assume that homozygotes $A A$ and $a a$ play the pure strategies $E_{1}$ and $E_{2}$, respectively, and that the heterozygote $A a$ plays the mixed strategy $\frac{1}{2}\left(E_{1}+E_{2}\right)$. In a similar way the $B$-locus determines female behavior: $B \rightarrow F_{1}=$ fast, $b \rightarrow F_{2}=$ coy. Let $p_{A}, q_{A}$ be the frequencies of allele $A$ in adult males and females, respectively, and $p_{B}, q_{B}$ be the corresponding frequencies of allele $B$.

Assuming linkage equilibrium, the frequencies of $A A, A a$, $a a$ in the next generation are $p_{A} q_{A}, p_{A}\left(1-q_{A}\right)+q_{A}\left(1-p_{A}\right)=p_{A}+q_{A}-2 p_{A} q_{A}$, $\left(1-p_{A}\right)\left(1-q_{A}\right)$, respectively. The marginal frequency of gene $A$, which also measures the probability of strategy $E_{1}$ among males in the new generation, is then given by $p_{A} q_{A}+\frac{1}{2}\left(p_{A}+q_{A}-2 p_{A} q_{A}\right)=\frac{1}{2}\left(p_{A}+q_{A}\right)$. Similar results hold for the $B$-locus.

In order to simplify the mathematical analysis, we replace Dawkins' original pay-off matrix (1) by the following more symmetric one (this does not change the situation qualitatively)

$$
A=\left(\begin{array}{ll}
1-K & 1+K \\
1+K & 1-K
\end{array}\right), \quad B=\left(\begin{array}{cc}
1+L & 1-L \\
1-L & 1+L
\end{array}\right)
$$

with $0<K, L<1$. This gives the following fitness values for males, depending on the frequency of females:

$$
\begin{aligned}
& w_{A A}=(1-K) \frac{p_{B}+q_{B}}{2}+(1+K)\left(1-\frac{p_{B}+q_{B}}{2}\right)=1+K\left(1-p_{B}-q_{B}\right) \\
& w_{a a}=(1+K) \frac{p_{B}+q_{B}}{2}+(1-K)\left(1-\frac{p_{B}+q_{B}}{2}\right)=1-K\left(1-p_{B}-q_{B}\right) \\
& w_{A a}=\frac{1}{2}\left(w_{A A}+w_{a a}\right)=1 .
\end{aligned}
$$

with similar results for females.

Hence writing $p_{A}^{\prime}$, etc., for the allelic frequencies in the adults of the new generation,

$$
\begin{aligned}
& p_{A}^{\prime}=\frac{w_{A A} p_{A} q_{A}+\frac{1}{2} w_{A a}\left(p_{A}+q_{A}-2 p_{A} q_{A}\right)}{\bar{w}_{A}} \\
& q_{A}^{\prime}=\frac{p_{A} q_{A}+\frac{1}{2}\left(p_{A}+q_{A}-2 p_{A} q_{A}\right)}{1}=\frac{p_{A}+q_{A}}{2}
\end{aligned}
$$


The mean fitness of males at the $A$-locus, $\bar{w}_{A}$, is given by

$$
\begin{aligned}
\bar{w}_{A} & =1+K\left[p_{A} q_{A}\left(1-p_{B}-q_{B}\right)-\left(1-p_{A}\right)\left(1-p_{B}-q_{B}\right)\right] \\
& =1-K\left(1-p_{A}-q_{A}\right)\left(1-p_{B}-q_{B}\right) .
\end{aligned}
$$

This leads finally to the equations

$$
\begin{aligned}
& p_{A}^{\prime}=\frac{\frac{1}{2}\left(p_{A}+q_{A}\right)+K\left(1-p_{B}-q_{B}\right) p_{A} q_{A}}{1-K\left(1-p_{A}-q_{A}\right)\left(1-p_{B}-q_{B}\right)} \\
& q_{A}^{\prime}=\frac{1}{2}\left(p_{A}+q_{A}\right) \\
& p_{B}^{\prime}=\frac{1}{2}\left(p_{B}+q_{B}\right) \\
& q_{B}^{\prime}=\frac{\frac{1}{2}\left(p_{B}+q_{B}\right)-L\left(1-p_{A}-q_{A}\right) p_{B} q_{B}}{1+L\left(1-p_{A}-q_{A}\right)\left(1-p_{B}-q_{B}\right)} .
\end{aligned}
$$

Since $0 \leqslant K, L<1$ this is a well-defined transformation on the state space $[0,1]^{4}$.

Obviously $p_{A}=q_{A}=p_{B}=q_{B}=\frac{1}{2}$ is a stationary solution of (7) with two zero eigenvalues (corresponding to a very quick convergence to a sex ratio $1: 1$ ) and a pair of complex conjugate eigenvalues with real part +1 (due to the cyclic structure of Dawkins' game) which make this solution unstable.

On the other hand, using the method of "average Ljapunov functions," developed by Hofbauer and Sigmund (1984) and Hutson and Moran (1982), one can analyze the local behavior near the boundary of the state space, showing that this boundary is repelling, and system (7) exhibits "permanent coexistence" (see Appendix A). The attractors of (7) thus have to lie in the interior of $[0,1]^{4}$, and since the only stationary solution there is unstable, this attractor has to be a more complicated set.

Numerical simulations show that this attractor is a "limit cycle," i.e., a closed invariant attracting curve surrounding the stationary solution (see Fig. 1). In the next section we will give a mathematical proof of this obser-

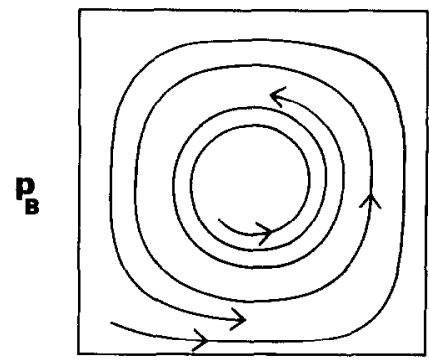

$\mathbf{P}_{\mathbf{A}}$

FIG. 1. The limit cycle. 
vation for small values of $L, K$ (this is the biological most relevant case) and determine the explicit equation of this limit cycle in the limit $K=L \rightarrow 0$. This is done by averaging techniques, as, for example, when treating Van der Pol's equation as a perturbation of the harmonic oscillator.

\section{The Limit Cycle}

We begin with a linear change of variables which transforms the stationary solution to the origin:

$x=1-p_{A}-q_{A}, \quad y=1-p_{B}-q_{B}, \quad u=p_{A}-q_{A}, \quad v=p_{B}-q_{B}$.

Then $(x, y, u, v) \in[-1,+1]^{4}$.

Furthermore we assume $K=L$ to make the analysis simpler and set $\varepsilon=K / 4$. Then $(7)$ is transformed into

$$
\begin{array}{ll}
x^{\prime}=x-\varepsilon y \frac{1-x^{2}-u^{2}}{1-4 \varepsilon x y}, & y^{\prime}=y+\varepsilon x \frac{1-y^{2}-v^{2}}{1+4 \varepsilon x y} \\
u^{\prime}=\varepsilon y \frac{1-x^{2}-u^{2}}{1-4 \varepsilon x y}, & v^{\prime}=-\varepsilon x \frac{1-y^{2}-v^{2}}{1+4 \varepsilon x y} .
\end{array}
$$

Now $\left|u^{\prime}\right|,\left|v^{\prime}\right| \leqslant \varepsilon /(1+4 \varepsilon)$, and so $u$ and $v$ will be of order $O(\varepsilon)$ for $\varepsilon \rightarrow 0$, uniformly on the whole state-space, in all subsequent generations. (This corresponds to a rough equilibration of the sex-ratio.) Thus the fourdimensional system (9) reduces for small $\varepsilon$ to a two-dimensional one,

$$
\begin{aligned}
& x^{\prime}=x+\varepsilon f(x, y)+\varepsilon^{2} f(x, y)+O\left(\varepsilon^{3}\right) \\
& y^{\prime}=y+\varepsilon g(x, y)+\varepsilon^{2} \tilde{g}(x, y)+O\left(\varepsilon^{3}\right)
\end{aligned}
$$

with

$$
\begin{array}{ll}
f(x, y)=-y\left(1-x^{2}\right), & \tilde{f}(x, y)=-4 x y^{2}\left(1-x^{2}\right) \\
g(x, y)=x\left(1-y^{2}\right), & \tilde{g}(x, y)=-4 x^{2} y\left(1-y^{2}\right) .
\end{array}
$$

For $\varepsilon \rightarrow 0$ we obtain, as a first-order approximation, the differential equation

$$
\begin{aligned}
& \dot{x}=f(x, y)=-y\left(1-x^{2}\right) \\
& \dot{y}=g(x, y)=x\left(1-y^{2}\right) .
\end{aligned}
$$

These are - up to a translation of the Nash solution to the origin-just the Schuster-Sigmund equations (3) for Dawkins' game, see also Maynard 
Smith (1982), Appendix J. Equations (12) coincide under our simplifying assumptions also with the Bomze et al. (1983) continuous-time genetic model.

These equations are integrable with

$$
I=R^{2}=x^{2}+y^{2}-x^{2} y^{2}
$$

as a constant of motion. Therefore we change to action-angle variables $(R, \varphi)$ such that (12) appears in the normal form (see Arnold, 1983)

$$
\dot{z}=i z \omega\left(|z|^{2}\right)=i\left(a_{0} z+a_{1} z|z|^{2}+a_{2} z|z|^{4}+\cdots\right)
$$

with $z=R e^{i \varphi}$, or equivalently

$$
\dot{R}=0, \quad \dot{\varphi}=\omega\left(R^{2}\right) .
$$

The canonical angle variable $\varphi$ and the angle velocity $\omega\left(R^{2}\right)$ are evaluated in Appendix B. They can be expressed only in terms of elliptic integrals.

Now we shall express our difference equation (10) in terms of the canonical variables $I=R^{2}$ and $\varphi$,

$$
\begin{aligned}
I^{\prime}= & I\left(x^{\prime}, y^{\prime}\right)=I\left(x+\varepsilon f+\varepsilon^{2} \tilde{f}+\cdots, y+\varepsilon g+\varepsilon^{2} \tilde{g}+\cdots\right) \\
= & I(x, y)+\left(\varepsilon f+\varepsilon^{2} \tilde{f}\right) I_{x}+\left(\varepsilon g+\varepsilon^{2} \tilde{g}\right) I_{y}+\frac{1}{2}\left[(\varepsilon f+\cdots)^{2} I_{x x}\right. \\
& \left.+2(\varepsilon f+\cdots)(\varepsilon g+\cdots) I_{x y}+(\varepsilon g+\cdots)^{2} I_{y y}\right]+O\left(\varepsilon^{3}\right) .
\end{aligned}
$$

Now the linear terms in $\varepsilon$ vanish since (14) implies $\dot{I}=I_{x} f+I_{y} g=0$. For the $\varepsilon^{2}$-terms we obtain from (13)

$$
I_{x}=2 x\left(1-y^{2}\right), \quad I_{x x}=2\left(1-y^{2}\right), \quad I_{x y}=-4 x y, \ldots
$$

Therefore

$$
I^{\prime}=I+\varepsilon^{2} F(x, y)+O\left(\varepsilon^{3}\right)
$$

with

$$
\begin{aligned}
F(x, y)= & f I_{x}+\tilde{g} I_{y}+\frac{1}{2}\left(f^{2} I_{x x}+2 f g I_{x y}+g^{2} I_{y y}\right) \\
= & -4 x y^{2}\left(1-x^{2}\right) 2 x\left(1-y^{2}\right)-4 x^{2} y\left(1-y^{2}\right) 2 y\left(1-x^{2}\right) \\
& +y^{2}\left(1-x^{2}\right)^{2}\left(1-y^{2}\right)+x y\left(1-x^{2}\right)\left(1-y^{2}\right) 4 x y \\
& +x^{2}\left(1-y^{2}\right)^{2}\left(1-x^{2}\right) \\
= & \left(1-x^{2}\right)\left(1-y^{2}\right)\left[-16 x^{2} y^{2}+y^{2}\left(1-x^{2}\right)+4 x^{2} y^{2}+x^{2}\left(1-y^{2}\right)\right] \\
= & \left(1-R^{2}\right)\left(x^{2}+y^{2}-14 x^{2} y^{2}\right) \\
= & \frac{1-R^{2}}{1-x^{2}}\left[R^{2}-14 R^{2} x^{2}+13 x^{4}\right] .
\end{aligned}
$$


Similarly, using $\dot{\varphi}=\varphi_{x} f+\varphi_{y} g=\omega(I)$, we obtain

$$
\begin{aligned}
\varphi^{\prime} & =\varphi\left(x^{\prime}, y^{\prime}\right)=\varphi(x+\varepsilon f+\cdots, y+\varepsilon g+\cdots) \\
& =\varphi(x, y)+\varepsilon f \varphi_{x}+\varepsilon g \varphi_{y}+O\left(\varepsilon^{2}\right) \\
& =\varphi+\varepsilon \omega\left(R^{2}\right)+O\left(\varepsilon^{2}\right) .
\end{aligned}
$$

So we end up with

$$
\begin{aligned}
I^{\prime} & =I+\varepsilon^{2} F(I, \varphi)+O\left(\varepsilon^{3}\right) \\
\varphi^{\prime} & =\varphi+\varepsilon \omega(I)+O\left(\varepsilon^{2}\right) .
\end{aligned}
$$

The next step is to average out the $\varphi$-dependence in the $\varepsilon^{2}$-term of (18) by the Ansatz $J=I+\operatorname{ch}(I, \varphi)$. Then

$$
\begin{aligned}
J^{\prime}= & I^{\prime}+\varepsilon h\left(I^{\prime}, \varphi^{\prime}\right)=I+\varepsilon^{2} F(I, \varphi)+\varepsilon h\left(I+\varepsilon^{2} F+\cdots, \varphi+\varepsilon \omega(I)+\cdots\right)+\cdots \\
& =I+\varepsilon h(I, \varphi)+\varepsilon^{2}\left[F(I, \varphi)+\frac{\partial h}{\partial \varphi} \omega(I)\right]+O\left(\varepsilon^{3}\right) .
\end{aligned}
$$

Now, following the usual averaging procedure, compare, e.g., Arnold (1983, p. $147 \mathrm{ff})$, we separate $F$ into its mean and its oscillating part,

$$
F(I, \varphi)=G(I)+\tilde{F}(I, \varphi)
$$

with

$$
G(I)=\frac{1}{2 \pi} \int_{0}^{2 \pi} F(I, \varphi) d \varphi .
$$

Inserting this into (19) we may choose $h$ in such a way that

$$
\tilde{F}(I, \varphi)+\frac{\partial h}{\partial \varphi} \omega(I)=0
$$

or

$$
\frac{\partial h}{\partial \varphi}=-\frac{\tilde{F}(I, \varphi)}{\omega(I)} .
$$

(Since $h$ is a $2 \pi$-periodic function in $\varphi$, the mean of its derivative, $(1 / 2 \pi) \int_{0}^{2 \pi}(\partial h / \partial \varphi) d \varphi$, equals zero. Since this will not in general be the case for the mean of $F(I, \varphi)$ we can average out only the oscillating part $\tilde{F}(I, \varphi)$.)

So with our new variable $J$ instead of $I$ we have simplified (18) to

$$
\begin{aligned}
J^{\prime} & =J+\varepsilon^{2} G(J)+O\left(\varepsilon^{3}\right) \\
\varphi^{\prime} & =\varphi+\varepsilon \omega(J)+O\left(\varepsilon^{2}\right) .
\end{aligned}
$$




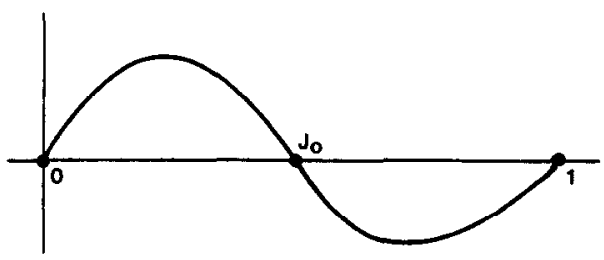

FIG. 2. Graph of the function $G(J)$.

The averaged function $G(J)$ is computed in Appendix B (in terms of elliptic integrals again), where we also prove the existence of a number $J_{0}$ such that $G(0)=G(1)=G\left(J_{0}\right)=0,(d / d J) G\left(J_{0}\right)<0$

$$
\begin{array}{lll}
G(J)>0 & \text { for } & 0<J<J_{0} \\
G(J)<0 & \text { for } & J_{0}<J<1 .
\end{array}
$$

Thus the graph of $G$ is similar to that shown in Fig. 2 .

If we neglect the $O$-terms in (22), then $J=J_{0}$ is obviously the equation of an invariant globally attracting circle. That this circle also persists under the $O$-perturbations for small $\varepsilon>0$, can be shown, e.g., by the technique developed by Iooss (1979) for proving the Hopf bifurcation theorem for maps.

In particular it is found that for small $\varepsilon>0,(10)$ has an attracting limit cycle of the form

$$
J=I+\operatorname{ch}(I, \varphi)=J_{0}+O\left(\varepsilon^{1 / 2}\right) .
$$

So one particular periodic orbit from the Hamiltonian system (12), i.e., the Schuster-Sigmund dynamics for Dawkins' game, $R^{2}=$ $x^{2}+y^{2}-x^{2} y^{2}=J_{0}$, serves as the limiting case $\varepsilon \rightarrow 0$ for the limit cycles of our more refined genetic model of the "battle of the sexes." The constant $J_{0}$ which determines the position of this cycle is the zero of the function $G(J)$ and is computed in Appendix B.

\section{Discussion}

We have discribed limit cycle behavior in a simple genetic model, with two alleles at each of two loci, with sex-dependence and fitnesses depending on the frequencies of the other sex. For small $\varepsilon$, measuring the intensity of frequency dependent selection, we have proved the existence and stability of a limit cycle. The model is based on Dawkins' (1976) "battle of the sexes" which is the simplest evolutionary game without an ESS.

This outcome-a globally stable limit cycle-would not be possible if the 
Nash equilibrium of the game were evolutionarily stable. If the phenotypic frequencies of an ESS, pure or mixed, correspond to a possible genetic state of the population, then that genetic state will be (locally) stable (in a continuous time dynamics or in discrete time with a small enough step-length). Although in this generality this is still an unproven statement it is strongly supported by the results of Maynard Smith (1981), Eshel (1982), Lessard (1984), Cressman and Hines (1984), Hines and Bishop (1984), and Thomas (1985).

Thus the results of the present paper supplement ESS theory and provide insight into what may happen for evolutionary games without ESS. Whereas at the game-theoretic level and even for haploid dynamics the situation is unclear and confusing the introduction of the appropriate underlying genetic structure clarifies the picture completely. Whereas the three haploid models mentioned in the Introduction, though nonlinear, essentially give rise to linear-like oscillatory behavior (conservative, damped, or biologically unreasonable divergent oscillations) the genetic model has the right nonlinearities to produce a both biologically interesting and mathematically nontrivial behavior: a globally stable limit cycle.

The amplitude of this limit cycle is rather insensitive to changes in the selection intensity, while the "period" is approximately inversely proportional to the selection intensity. This is in contrast to limit cycle behavior constructed by standard Hopf bifurcation techniques where the amplitude is small and the period is approximately constant.

In the limit $\varepsilon \rightarrow 0$, this limit cycle tends to one particular cycle of the simple Hamiltonian system identified by Schuster and Sigmund (1981) as the first dynamic model for Dawkins' game. In particular, at least for $\varepsilon \rightarrow 0$, the time average over the limit cycle represents the interior stationary solution predicted by game theory. Thus this solution, though unstable both dynamically and evolutionarily, still has some biological relevance. This shows once more that despite their simplicity such haploid models already carry the basic ingredients.

Numerical simulations show that this limit cycle behavior is rather robust. It does not depend on the assumption that fitnesses are additive, or that the selection intensity is the same in the two sexes (i.e., $K=L$ ). Limit cycle behavior is still observed if there is some directional, non-frequencydependent selection, provided that this directional selection is less intense than the frequency-dependent selection. Also, the conclusion does not depend on the assumption that the genes are sex-limited in their effects. A similar limit cycle arises if the fitnesses of the genotypes at the $A$ locus, in both sexes, depend additively on the frequencies of the alleles at the $B$ locus, and vice versa.

All these observations could be proved using the same method of averaging. We have confined ourselves to the simplest possible case, 
however, in order to keep the mathematical analysis tractable. In more general models difficulties could arise since the nice elliptic integrals have to be replaced by more general integrals where formulas like (B5-7) are probably not available. S. van Gils (personal communication) has recently obtained another different proof of the existence and stability of the limit cycle which avoids the explicit computation of the elliptic integrals and uses Picard-Fuchs equations instead. We expect that his technique will be helpful to treat the more general cases.

That limit cycle behavior is possible in rather simple genetic sysems has been shown in the last few years. The pioneering work in this direction is due to Akin (1979). Other papers dealing with this topic are Akin (1982, 1983), Hastings (1981), Hofbauer and Iooss (1984), Hofbauer $(1984,1985)$, Hunt (1982), Koth and Kemler (1985), Selgrade and Namkoong (1984).

\section{APPENDIX A: BEHAVIOR NEAR THE BOUNDARY}

First it is easy to check that one of $p_{A}^{\prime}, q_{A}^{\prime}, p_{B}^{\prime}, q_{B}^{\prime}$ is zero if and only if one of the four alleles $A, a, B, b$ is missing in both sexes. In the new variables (8): $x= \pm 1$ or $y= \pm 1$. Thus the set

$$
M=\{|x|=1 \text { or }|y|=1\},
$$

which is a union of four squares, is the maximal invariant subset of the boundary of the state space: Starting on the boundary outside $M$, the next generation and all subsequent ones will be in the interior of $[0,1]^{4}$, i.e., all four alleles will be present in both sexes. Thus in order to show that the boundary is a repelling set, it is sufficient to prove that $M$ is repelling.

For this we first need the behavior on $M$ itself. Since $x^{\prime} \leqslant x$ iff $y \geqslant 0$, it is easy to see that on the part where $y=-1, x$ converges monotonically, increasing from -1 to 1 . Where $x=1, y$ goes from -1 to 1 ; where $y=1, x$ goes from 1 back to -1 , and finally where $x=-1, y$ decreases from 1 to -1 , and the cycle is closed. Thus the $\omega$-limit set of $M$ consists only of the four fixed points where $|x|=|y|=1$ (and $u=v=0$ ).

Now consider the function

$$
P=\left(1-x^{2}\right)\left(1-y^{2}\right) .
$$

Then $P \geqslant 0$ holds, with $P=0$ on $M$. Furthermore $P^{\prime} / P>\delta>0$ on the whole state and space and

$$
\frac{P^{\prime}}{P}=\left(\frac{1-4 \varepsilon^{2}}{1-16 \varepsilon^{2}}\right)^{2}>1
$$


at the four fixed points in $M$. Thus $P^{\prime} / P>1$ on the whole $\omega$-limit set of $M$, and $P$ is an "average Lyapunov function" for $M$. According to Hutson and Moran (1982), $M$ is a repeller.

\section{APPENDIX B: Elliptic Integrals in the Battle of the SeXes}

We start with the normal form (14)

$$
\dot{R}=0, \quad \dot{\varphi}=\omega\left(R^{2}\right)
$$

of the Schuster-Sigmund differential equation (12) for the battle of the sexes. Since, by (13), we have

$$
R^{2}=x^{2}+y^{2}-x^{2} y^{2}
$$

the state space $\left\{(x, y) \in \mathbb{R}^{2}:|x|<1\right.$ and $\left.|y|<1\right\}$ for (12) transforms into $\{(R, \varphi) \in \mathbb{R} \times \mathbb{R} / 2 \pi \mathbb{Z}: 0 \leqslant R<1\}$. In order to determine the canonical angle $\varphi$ and the angle velocity $\omega\left(R^{2}\right)$ we treat $\varphi$ as a function of $x$ and $R: \varphi=\varphi(x, R)$. Then $\dot{\varphi}=\varphi_{x} \dot{x}+\varphi_{R} \dot{R}$ implies

$$
\omega\left(R^{2}\right)=-\varphi_{x} y\left(1-x^{2}\right) .
$$

Eliminating $y$ from (B2),

$$
\frac{d \varphi}{d x}=-\omega\left(R^{2}\right)\left(1-x^{2}\right)^{-1 / 2}\left(R^{2}-x^{2}\right)^{-1 / 2} .
$$

It is well known that this leads to an elliptical integral. In particular we obtain

$$
-\int_{-R}^{+R} \varphi_{x} d x=-\left.\varphi\right|_{-R} ^{+R}=\pi=\omega\left(R^{2}\right) \int_{-R}^{+R}\left(1-x^{2}\right)^{-1 / 2}\left(R^{2}-x^{2}\right)^{-1 / 2} d x .
$$

Now recall Legendre's formulas for complete elliptic integrals (see, e.g., Groebner \& Hofreiter, 1950, p. 39)

$$
\begin{aligned}
\int_{0}^{R}\left(1-x^{2}\right)^{-1 / 2}\left(R^{2}-x^{2}\right)^{-1 / 2} d x & =\int_{0}^{1}\left(1-R^{2} x^{2}\right)^{-1 / 2}\left(1-x^{2}\right)^{-1 / 2} d x \\
& =K(R)=\frac{\pi}{2} \sum_{n=0}^{\infty}\left(\begin{array}{c}
1 / 2 \\
n
\end{array}\right)^{2} R^{2 n}
\end{aligned}
$$




$$
\begin{aligned}
\int_{0}^{R}\left(1-x^{2}\right)^{1 / 2}\left(R^{2}-x^{2}\right)^{-1 / 2} d x & =\int_{0}^{1}\left(1-R^{2} x^{2}\right)^{1 / 2}\left(1-x^{2}\right)^{-1 / 2} d x \\
& =E(R)=\frac{\pi}{2} \sum_{n=0}^{\infty}\left(\begin{array}{c}
-1 / 2 \\
n
\end{array}\right)\left(\begin{array}{c}
1 / 2 \\
n
\end{array}\right) R^{2 n} \\
\int_{0}^{R}\left(1-x^{2}\right)^{-3 / 2}\left(R^{2}-x^{2}\right)^{-1 / 2} d x & =\int_{0}^{1}\left(1-R^{2} x^{2}\right)^{-3 / 2}\left(1-x^{2}\right)^{-1 / 2} d x \\
& =\left(1-R^{2}\right)^{-1} E(R),
\end{aligned}
$$

where $\left(\begin{array}{l}\alpha \\ n\end{array}\right)=\alpha(\alpha-1) \cdots(\alpha-n+1) / n$ ! is the binomial coefficient. These series converge for $|R|<1$. Equations (B4) and (B5) imply

$$
\omega\left(R^{2}\right)=\frac{\pi}{2 K(R)}=\left(\sum_{n=0}^{\infty}\left(\begin{array}{c}
1 / 2 \\
n
\end{array}\right)^{2} R^{2 n}\right)^{-1} .
$$

The next problem is to evaluate the averaged function (20):

$$
G\left(R^{2}\right)=\frac{1}{2 \pi} \int_{0}^{2 \pi} F\left(R^{2}, \varphi\right) d \varphi .
$$

Since we do not have an explicit expression for $\varphi$, we have to transform the integral. Using (B3) and splitting the circle into 4 equal parts we obtain

$$
\begin{aligned}
G\left(R^{2}\right) & =\frac{2 \omega\left(R^{2}\right)}{\pi} \int_{0}^{R} \frac{F\left(R^{2}, x\right)}{y\left(1-x^{2}\right)} d x \\
& =\frac{2}{\pi} \omega\left(R^{2}\right) \int_{0}^{R} F\left(R^{2}, x\right)\left(R^{2}-x^{2}\right)^{-1 / 2}\left(1-x^{2}\right)^{-1 / 2} d x .
\end{aligned}
$$

So again we have to calculate an elliptic integral. From (16) we obtain

$$
\begin{aligned}
(1- & \left.R^{2}\right)^{-1} \int_{0}^{R} F\left(R^{2}, x\right)\left(1-x^{2}\right)^{-1 / 2}\left(R^{2}-x^{2}\right)^{-1 / 2} d x \\
= & \int_{0}^{R}\left(R^{2}-14 R^{2} x^{2}+13 x^{4}\right)\left(1-x^{2}\right)^{-3 / 2}\left(R^{2}-x^{2}\right)^{-1 / 2} d x \\
= & 13 \int_{0}^{R}\left(1-x^{2}\right)^{1 / 2}\left(R^{2}-x^{2}\right)^{-1 / 2} d x \\
& +\left(14 R^{2}-26\right) \int_{0}^{R}\left(1-x^{2}\right)^{-1 / 2}\left(R^{2}-x^{2}\right)^{1 / 2} d x \\
& +13\left(1-R^{2}\right) \int_{0}^{R}\left(1-x^{2}\right)^{-3 / 2}\left(R^{2}-x^{2}\right)^{-1 / 2} d x \\
= & 13 E(R)+\left(14 R^{2}-26\right) K(R)+13 E(R) \\
= & 26 E(R)+\left(14 R^{2}-26\right) K(R) .
\end{aligned}
$$


So (B10) together with (B8) gives an "explicit" formula for $G$,

$$
G\left(R^{2}\right)=\left(1-R^{2}\right)\left(14 R^{2}-26+26 \frac{E(R)}{K(R)}\right) .
$$

Inserting the series expansions (B5), (B6) into (B11) we obtain after some calculation

$$
G\left(R^{2}\right)=\omega\left(R^{2}\right) R^{2}\left(1-R^{2}\right) g\left(R^{2}\right)
$$

with

$$
\begin{aligned}
g(x) & =\sum_{n=0}^{\infty} \frac{1}{n+1}\left(-52 n^{2}+66 n+1\right)\left(\begin{array}{c}
1 / 2 \\
n
\end{array}\right)^{2} x^{n} \\
& =1+\frac{15}{8} x-\frac{25}{64} x^{2}-\cdots
\end{aligned}
$$

Obviously all terms in the series $g(x)$-up to the first two-have a negative coefficient. Hence $d^{2} g(x) / d x^{2}<0$ for $0<x<1$. Moreover, $\left.G\left(R^{2}\right)\left(1-R^{2}\right)^{-1}\right|_{R=1}=-12<0$ from (B12) implies $g(1)=-\infty$. Since $g(0)=1$, the function $g(x)$ has a unique zero $J_{0}$ in the interval $(0,1)$ and $G\left(R^{2}\right)$ is therefore of the desired form (23). The numerical value of $J_{0}$ can be found approximately from a table of elliptic functions $J_{0} \cong 0.46$.

\section{ACKNOWLEDGMENT}

We thank Professor Karl Sigmund for his advice and encouragement during the preparation of this paper.

\section{REFERENCES}

AkIN, E. 1979. "The Geometry of Population Genetics," Lecture Notes in Biomathematics, Vol. 31, Springer, Berlin.

AKIN, E. 1982. Cycling in simple genctic systems, J. Math. Biol. 13, 305-324.

Akin, E. 1983. Hopf bifurcation in the two locus genetic models, in "Memo. Amer. Math. Soc.," No. 284, Providence, R.I.

ArNold, V. I. 1983. "Geometrical Methods in the Theory of Ordinary Differential Equations," Grundlehren Math. Wissenschaften, Vol. 250, Springer, Berlin.

Bomze, I., Schuster, P., AND Sigmund, K. 1983. The role of Mendelian genetics in strategic modeis on animal behavior, J. Theor. Biol. 101, 19-38.

Cressman, R., and Hines, W. G. S. 1984. Evolutionarily stable strategies of diploid populations with semi-dominant inheritance patterns, J. Appl. Prob. 21, 1-9.

Dawkins, R. 1976. "The Selfish Gene," Oxford Univ. Press, Oxford.

ESHEL. I. 1982. Evolutionarily stable strategies and viability selection in Mendelian populations, Theor. Pop. Biol. 25, 204-217. 
Eshel, I., AND AKIN, E. 1983. Coevolutionary instability of mixed Nash solutions, J. Math. Biol. 18, 123-133.

Groebner, W., AND Hofreiter, N. 1950. "Integraltafel, 2. Teil: Bestimmte Integrale," Springer, Berlin.

HaStings, A. 1981. Stable cycling in discrete-time genetic models, Proc. Natl. Acad. U.S.A. 11, 7224-7225.

HiNES, W. G. S., AND BiSHOP, D. T. 1984. Can and will a sexual diploid population attain an evolutionary stable strategy? J. Theor. Biol. 111, 667-686.

HofBaUER, J. 1984. Gradients versus cycling in genetic selection models, in "Dynamics of Macrosystems, Proc. Laxenburg 1984" (J.-P. Aubin, D. Saari, K. Sigmund, Eds.), Lecture Notes in Economics and Mathematical Systems, Vol. 257, pp. 90-101, Springer, Berlin.

Hofbauer, J. 1985. The selection mutation equation, J. Math. Biol. 23, 41-53.

HoFBaUer, J. 1987. Are there mixed ESS in asymmetric games? To appear.

Hofbauter, J. ANd Iooss, G. 1984. A Hopf bifurcation theorem for difference equations approximating a differential equation, Monatsh. Math. 98, 99-113.

Hofbauer, J. AND Sigmund, K. 1984. "Evolutionstheorie und dynamische Systeme. Mathematische Aspekte der Selektion," Parey, Hamburg/Berlin.

HuNT, F. 1982. Regulation of population cycles by genetic feedback: Existence of periodic solutions of a mathematical model, J. Math. Biol. 13, 271-282.

Hutson, V., AND MORAN, W. 1982. Persistence of species obeying difference equations, J. Math. Biol. 15, 203-213.

Iooss, G. 1979. "Bifurcations of Maps and Applications," North-Holland Math. Studies, Vol. 36, North-Holland, Amsterdam.

Koth, M., AND Kemler, F. 1986. A one locus-two allele selection model admitting stable limit cycles, J. Theor. Biol. 122, 263-267.

LESSARD, S. 1984. Evolutionary dynamics in frequency-dependent two-phenotype models, Theor. Pop. Biol. 25, 210-234.

MaYNaRd Smith, J. 1981. Will a sexual population evolve to an ESS? Amer. Nat. 177, 1015-1018.

Maynard Smith, J. 1982. "Evolution and the Theory of Games," Cambridge Univ. Press, Cambridge.

SChuster, P., AND Sigmund, K. 1981. Coyness, philandering and stable strategies, Animal Behav. 29, 186-192.

Selokade, J. F., and Namkoong, G. 1984. Dynamical behavior of differential equation models of frequency and density dependent populations, J. Math. Biol. 19, 133-146.

SELTEN, R. 1980. A note on evolutionarily stable strategies in asymmetrical animal conflicts, J. Theor. Biol. 84, 93-101.

Thomas, B. 1985. Genetical ESS-models II. Multistrategy models and multiple alleles, Theor. Pop. Biol. 28, 33-49.

Zeeman, E. C., 1980. Population dynamics from game theory, in "Global Theory of Dynamical Systems," Lecture Notes in Mathematics, Vol. 819, Springer, Berlin. 